Technological University Dublin

DÜBLIN

ARROW@TU Dublin

2012-7

\title{
Chalcogenide Microsphere Fabricated from Fiber Tapers Using Contact With a High-Temperature Ceramic Surface
}

\author{
Pengfei Wang \\ Technological University Dublin, pengfei.wang@tudublin.ie \\ Ganapathy Murugan \\ University of Southampton \\ Gilberto Bramilla \\ University of Southampton
}

See next page for additional authors

Follow this and additional works at: https://arrow.tudublin.ie/engscheceart

Part of the Electromagnetics and Photonics Commons

\section{Recommended Citation}

Wang, P., Murugan, G., Brambilla, G., Ding, M., Semenova, Y., Wu, Q., Farrell, G.: Chalcogenide Microsphere Fabricated from Fiber Tapers Using Contact With a High-Temperature Ceramic Surface. IEEE Photonics Technology Letters, Vol. 24, 13, 2012, pp.1103-1105. doi:10.1109/LPT.2012.2195722

This Article is brought to you for free and open access by the School of Electrical and Electronic Engineering at ARROW@TU Dublin. It has been accepted for inclusion in Articles by an authorized administrator of ARROW@TU Dublin. For more information, please contact arrow.admin@tudublin.ie, aisling.coyne@tudublin.ie, gerard.connolly@tudublin.ie.

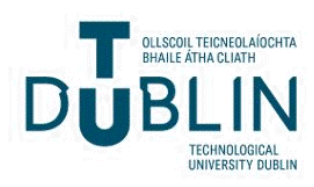




\section{Authors}

Pengfei Wang, Ganapathy Murugan, Gilberto Bramilla, Ming Ding, Yuliya Semenova, Qiang Wu, and Gerald Farrell 


\title{
Chalcogenide Microsphere Fabricated from Fiber Tapers Using Contact With a High-Temperature Ceramic Surface
}

\author{
Pengfei Wang, Ganapathy Senthil Murugan, Gilberto Brambilla, Ming Ding, \\ Yuliya Semenova, Qiang Wu, and Gerald Farrell
}

\begin{abstract}
The fabrication and characterization of a chalcogenide glass microsphere resonator has been demonstrated. At wavelengths near $1550 \mathrm{~nm}$, whispering gallery mode resonances can be efficiently excited in a 74- $\mu \mathrm{m}$-diameter chalcogenide glass microsphere via evanescent coupling using a tapered silica glass fiber with a waist diameter of circa $2 \mu \mathrm{m}$. Resonances with Q-factors greater than $10^{5}$ were observed. Due to the high nonlinearity properties of the chalcogenide material and the ease of fabrication process, chalcogenide microspheres offer the potential for robustly assembled fully integrated photonic devices.
\end{abstract}

Index Terms-Chalcogenide glass, fiber taper, microshpere, resistive heating.

\section{INTRODUCTION}

$\mathbf{O}$ VER the last decade interest in microsphere resonators has increased rapidly due to their very high Q-factors, the ease with which they can be manufactured and their versatility in terms of materials and dopants for a variety of passive and active devices. Furthermore, microsphere resonators have the potential to add significant functionality to planar lightwave circuits when coupled to waveguides where they can provide a range of unique functions, such as nonlinear optics, all-optical switching, wavelength filtering and lasing functions [1-4].

Chalcogenides are rapidly establishing themselves as technologically superior materials for emerging application in nonvolatile memory and high speed switching [5] and have been
Manuscript received August 13, 2011; revised February 18, 2012; accepted March 8, 2012. The work of P. Wang was supported in part by the Irish Research Council for Science, Engineering and Technology and in part by the European Union Marie-Curie Actions under FP7. The work of G. Brambilla was supported in part by the Royal Society, London, under a research fellowship. The work of Q. Wu was supported in part by Science Foundation Ireland under Grant 07/SK/I1200.

P. Wang is with the Optoelectronics Research Centre, University of Southampton, Southampton SO17 1BJ, U.K., and also with the Photonic Research Centre, Dublin Institute of Technology, Dublin 1, Ireland (e-mail: pw3y09@orc.soton.ac.uk).

G. S. Murugan, G. Brambilla, and M. Ding are with the Optoelectronics Research Centre, University of Southampton, Southampton SO17 1BJ, U.K. (e-mail: smg@orc.soton.ac.uk; gb2@orc.soton.ac.uk; md20d09@orc.soton.ac.uk).

Y. Semenova, Q. Wu, and G. Farrell are with the Photonic Research Centre, Dublin Institute of Technology, Dublin 1, Ireland (e-mail: yuliya.semenova@dit.ie; qiang.wu@dit.ie; gerald.farrell@dit.ie).

Color versions of one or more of the figures in this letter are available online at http://ieeexplore.ieee.org.

Digital Object Identifier 10.1109/LPT.2012.2195722 considered for a range of other optoelectronic technologies. Chalcogenide glasses offer a wealth of active properties such as exceptionally high nonlinearity, photosensitivity, low phonon energy matrix, the ability to be doped with active elements including lanthanides and transitional metals and the possibility to form detectors, lasers and amplifiers. Chalcogenides also display semiconductor, optical, acousto-optic, superconducting and opto-mechanical properties. Unlike any other optical material, they have been formed in to a multitude of shapes, including optical fibers, thin films, bulk optical components, microsphere resonators, metamaterials and nanoparticles, patterned by CMOS compatible processing at the sub micron scale.

To date, most studies on microsphere resonators have utilized silica microspheres fabricated by melting the tip of an optical fiber with the resulting stem attached to the microsphere used as a tool to place the sphere in the required location while characterizing the microsphere [6]. For fabricating a chalcogenide microshpere, several fabrication methods have been introduced recently [7-9], such as rapid quenching of liquid droplets [7], optical fusing in a tapered chalcogenide fiber [8] and a three-step heating process [9]. In this letter high quality chalcogenide $\left(\mathrm{As}_{2} \mathrm{~S}_{3}\right)$ microspheres with diameters as small as $74 \mu \mathrm{m}$ are directly fabricated from a simple taper-draw using contact with a high temperature ceramic surface. A relatively high quality factor greater than $10^{5}$ near a wavelength of $1550 \mathrm{~nm}$ is demonstrated with efficient coupling using a silica fiber taper with a diameter of $\sim 2 \mu \mathrm{m}$. The chalcogenide microspheres fabricated by using contact with a high temperature ceramic surface offer the potential for low-cost, robustly assembled fully integrated all-optical switching devices due to their unique high nonlinearity and ease of fabrication.

\section{FABrication of Chalcogenide Microsphere Using Resistive Heating Method}

The chalcogenide fiber used in the experiments is a commercial step-index multimode fiber provided by Oxford Electronics, with an $\mathrm{As}_{2} \mathrm{~S}_{3}$ core $(\mathrm{OD}=180 \mu \mathrm{m})$ and $\mathrm{As}_{x} \mathrm{~S}_{1-x}$ cladding of lower refractive index $(\mathrm{OD}=275 \mu \mathrm{m})$. Our approach to directly fabricating a chalcogenide microsphere is illustrated in Fig 1. As shown in the figure, firstly, a microheater is used to heat the chalcogenide fiber $\left(\right.$ at $\sim 200{ }^{\circ} \mathrm{C}$ ) 


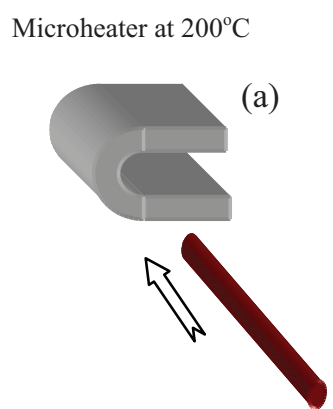

Microheater at $200^{\circ} \mathrm{C}$

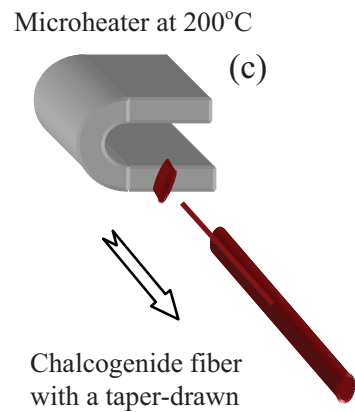

Microheater at $500^{\circ} \mathrm{C}$

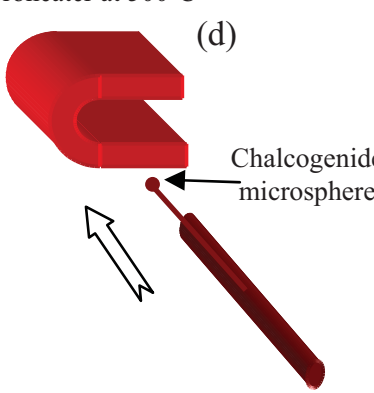

Fig. 1. Schematic diagram illustrating the fabrication process of a microsphere from chalcogenide fiber. (a) Chalcogenide fiber is moved towards a microheater set at a temperature of $200{ }^{\circ} \mathrm{C}$. (b) Fiber end touches the surface of the microheater and experiences local melting. (c) Portion of molten glass is left on the surface of the microheater and a tapered microfiber is formed at the end of the fiber when the fiber is withdrawn. (d) Microsphere is formed at the freestanding side of the taper.

and soften its end (Fig. 1a). When the fiber touches the surface of the outer wall of the microheater (Fig. 1b) it softens and adheres to the microheater surface; by then withdrawing the fiber at a speed of $0.1 \sim 1 \mathrm{~m} / \mathrm{s}$, the fiber tapers until breakage occurs (Fig. 1c). When the process is finished, a microfiber with a considerable length is formed at the freestanding side of the chalcogenide fiber. In the last step in the fabrication process, the microsphere is fabricated by bringing the tip of the tapered microfiber close to the microheater and heating the tip at a temperature higher than the transition temperature of chalcogenide material (Fig. 1d), around $500{ }^{\circ} \mathrm{C}$, so that surface tension pulls the melted glass tip into a spherical shape, thereby creating a microsphere resonator on the tip of the tapered microfiber. Experimentally it is found that the size of the microsphere fabricated using this method primarily varies with a number of experimental parameters, such as the diameter of the tapered fiber waist, the temperature of the microheater and the movement speed of the fiber taper towards the microheater.

Fig. 2(a) shows a taper with a microsphere at the end of it, while Fig. 2(b)-(d) show a microscopic top view of three chalcogenide glass microsphere resonators with diameters of $74 \mu \mathrm{m}, 98 \mu \mathrm{m}$ and $109 \mu \mathrm{m}$, respectively.

\section{Measurement And Analysis of the FABRICATED CHALCOGENIDE MicRosPHERES}

The experimental apparatus used for optical characterization of the chalcogenide microsphere is shown in Fig. 3. Light from a narrow-line tunable laser source (Agilent 81600B,

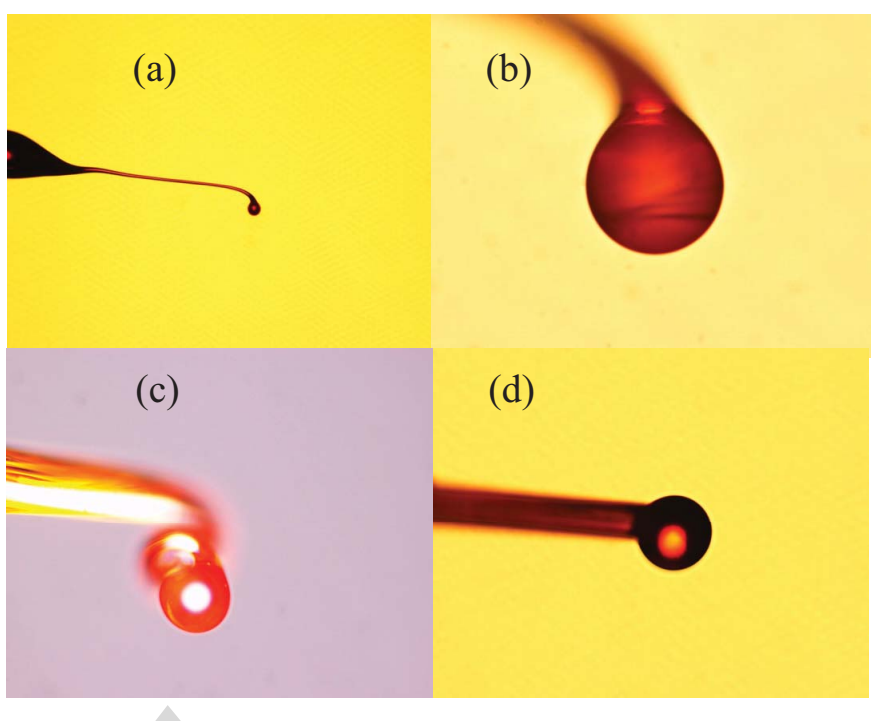

Fig. 2. (a) Microscope image of a chalcogenide fiber with a taper-drawn and a microsphere at the end of taper, three chalcogenide microspheres fabricated on the tapers showing good surface quality and diameters, (b) $74 \mu \mathrm{m}$, (c) $98 \mu \mathrm{m}$, and (d) $109 \mu \mathrm{m}$.

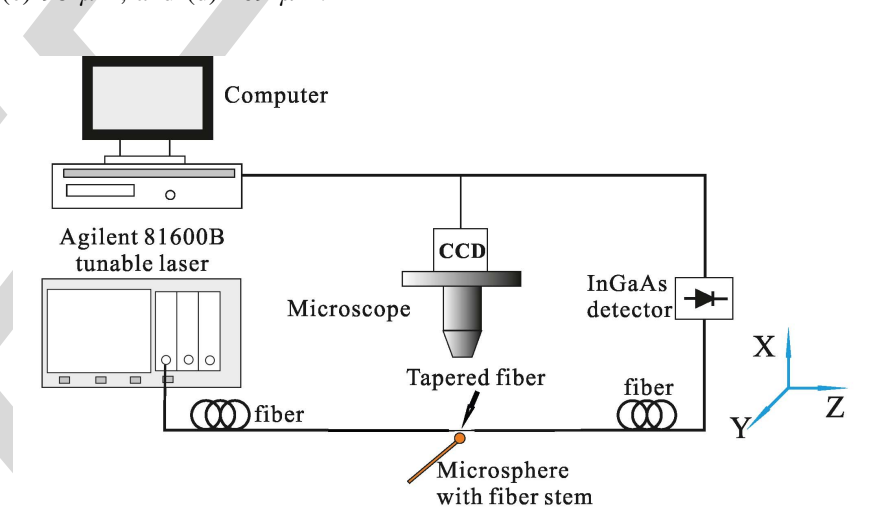

Fig. 3. Experimental apparatus used for chalcogenide microsphere resonance characterization.

power range: $\pm 7 \mathrm{dBm}$ ) emitting a power of $0 \mathrm{dBm}$ over the wavelength range $1540 \mathrm{~nm}$ to $1560 \mathrm{~nm}$, the input signal was launched into a tapered silica fiber and coupled to the chalcogenide microsphere. The throughput signal was collected using an InGaAs photodetector. The separation between the microsphere and the tapered fiber was controlled with a precision nanotranslation stage equipped with piezoelectric actuators and stepper motors and monitored using a microscope equipped with a CCD camera. A standard singlemode silica fiber was tapered as a coupling waveguide using the modified "flame brushing technique" [10] and the fiber taper with an extremely uniform waist diameter $(\mathrm{d} \sim 2 \mu \mathrm{m})$, the taper transitions of well defined length and shape were then made, the transmission loss of tapered fiber can reach levels lower than $0.1 \mathrm{~dB}$. The tapered fiber stem supporting the chalcogenide microsphere ensured that the chalcogenide microsphere orientation remained fixed with respect to the tapered silica coupling fiber as it was translated across and away from it.

Fig. 4 shows the top view of chalcogenide glass microsphere resonator with a diameter of $74 \mu \mathrm{m}$, in close proximity to a tapered silica fiber with a waist diameter $\mathrm{d} \sim 2 \mu \mathrm{m}$, and used 


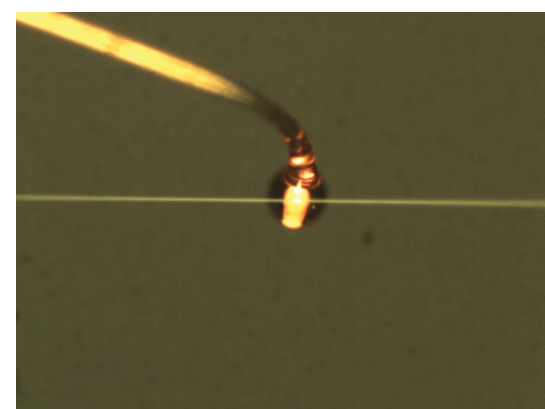

Fig. 4. Microscope images of chalcogenide microspheres with a diameter of $74 \mu \mathrm{m}$ showing its fiber stem and the tapered coupling silica fiber.

the well-established evanescent field coupling technique [10] The power transmitted through the excitation fiber taper was recorded as a function of wavelength at input power of $0 \mathrm{dBm}$. In this study, we used a tapered silica fiber instead of a tapered high index fiber to excite WGMs, so that any nonlinear effects from the delivery fiber can be minimized. This is reasonable since if the microspheres are used as the basis of a nonlinear photonic device it will be necessary to localize the nonlinear interactions within the microresonators and not in the signal delivery fibers.

Fig. 5 (bottom part) shows the transmission spectrum over a short wavelength range $1555 \sim 1555.25 \mathrm{~nm}$ outputted by the tapered silica fiber used as a coupling waveguide, showing the high- $Q$ nature of the observed resonance dips: a FWHM of $\sim 14 \mathrm{pm}$ was found, corresponding to a $Q$-factor of $\sim 1.1 \times 10^{5}$. The highest $\mathrm{Q}$ factor found here is from the "best defined" mode in the WGM spectra over a range of 1540-1560 nm. Note that in the experiments, the gap between the microsphere and the tapered fibre needs to be carefully adjusted, as any variation of the gap between the microsphere and the tapered fiber will necessarily induce not only variation of the coupling efficiency but also shift of the WGM resonance wavelengths [11]. In Ref. [9], a high- $Q$ factor greater than $2 \times 10^{6}$ has been observed due to the effective coupling using a high refractive index silicon nanowire in which can provide an appropriate phase matching between the silicon nanowire and the chalcogenide microsphere. In this letter a reasonably high- $Q$ of $1.1 \times 10^{5}$ in the telecommunications wavelength window has been demonstrated using a conventional silica taper as a coupling waveguide, which offers a robust coupling platform and easy integration into both on-chip and fiber systems. On the other hand, this fabrication process is very simple and has the benefit of being low-cost. Chalcogenide microsphere resonators are potential candidates to achieve alloptical processing of high-speed data in the telecommunication window, low-threshold supercontinuum generation, quantum cascade laser and related microsphere lasing devices. Efforts are underway to package the chalcogenide microsphere with a silica fiber taper using UV-curable resins to make a robust integrated device performing the above mentioned functions [12].

\section{CONCLUSION}

In conclusion, the fabrication of chalcogenide microspheres heated by contact with a temperature controlled ceramic

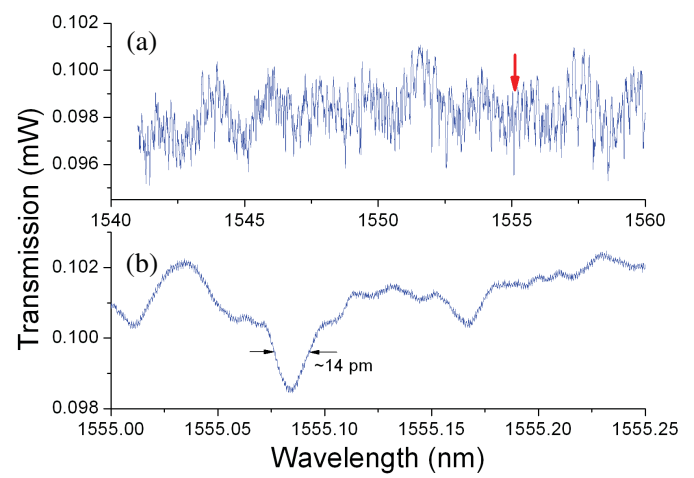

Fig. 5. One of the measured resonance dips near $1555 \mathrm{~nm}$. (a) Experimental resonance spectrum for wavelengths between 1541 and $1560 \mathrm{~nm}$ for the chalcogenide microsphere with a diameter of $74 \mu \mathrm{m}$, coupled with a tapered silica fiber. (b) Experimental resonance spectrum in the region of 1555-1555.25 $\mathrm{nm}$.

surface has been demonstrated. Whispering gallery mode resonances using tapered silica glass fiber as a signal delivery waveguide have been observed and a $Q$ factor greater than $10^{5}$ was recorded at $\lambda \sim 1.55 \mu \mathrm{m}$. Compared to the fabrication method presented in the Ref. [9], our method has several advantages, such as a higher $\mathrm{Q}$ factor and broad operation range due to lower surface oxidation. We believe that this work will provide a simple fabrication technique for chalcogenide material based microresonators as an ideal candidate for photonics building-blocks for several applications including highly integrated optical switches, modulators, ultrasmall optical filters and integrated microlasers.

\section{REFERENCES}

[1] S. M. Spillane, T. J. Kippenberg, and K. J. Vahala, "Ultralow-threshold Raman laser using a spherical dielectric microcavity," Nature, vol. 415, pp. 621-623, Feb. 2002.

[2] V. R. Almeida, C. A. Barrios, R. R. Panepucci, and M. Lipson, "Alloptical control of light on a silicon chip," Nature, vol. 431, pp. 10811084, Oct. 2004.

[3] S. Xiao, M. H. Khan, H. Shen, and M. Qi, "Multiple-channel silicon micro-resonator based filters for WDM applications," Opt. Express, vol. 15, no. 12, pp. 7489-7498, 2007.

[4] G. S. Murugan, M. N. Zervas, Y. Panitchob, and J. S. Wilkinson, "Integrated Nd-doped borosilicate glass microsphere laser," Opt. Lett., vol. 36, no. 1, pp. 73-75, 2011.

[5] B. J. Eggleton, B. Luther-Davies, and K. Richardson, "Chalcogenide photonics," Nature Photon., vol. 5, pp. 141-148, Feb. 2011.

[6] M. L. Gorodetsky, A. A. Savchenkov, and V. S. Ilchenko, "Ultimate Q of optical microsphere resonators," Opt. Lett., vol. 21, no. 7, pp. 453-455, 1996.

[7] G. R. Elliott, D. W. Hewak, G. S. Murugan, and J. S. Wilkinson, "Chalcogenide glass microspheres; their production, characterization and potential," Opt. Express, vol. 15, no. 26, pp. 17542-17553, 2007.

[8] C. Grillet, S. N. Bian, E. C. Magi, and B. J. Eggleton, "Fiber taper coupling to chalcogenide microsphere modes," Appl. Phys. Lett., vol. 92, no. 17 , pp. 171109-171111, 2008.

[9] D. H. Broaddus, M. A. Foster, I. H. Agha, J. T. Robinson, M. Lipson, and A. L. Gaeta, "Silicon-waveguide-coupled high-Q chalcogenide microspheres," Opt. Express, vol. 17, no. 8, pp. 5998-6003, 2009.

[10] G. Brambilla, F. Koizumi, X. Feng, and D. J. Richardson, "Compoundglass optical nanowires," Electron. Lett., vol. 41, no. 7, pp. 400-402, 2005.

[11] Z. Guo, H. Quan, and S. Pau, "Near-field gap effects on small microcavity whispering-gallery mode resonators," J. Phys. D, vol. 39, no. 24, pp. 5133-5136, 2006.

[12] Y.-Z. Yan, et al., "Packaged silica microsphere-taper coupling system for robust thermal sensing application," Opt. Express, vol. 19, no. 7, pp. 5753-5759, 2011. 